
\title{
R Research S Suare \\ SARS-CoV-2 Omicron infection is associated with high nasopharyngeal viral load
}

Gian Luca Salvagno

Brandon M. Henry

Laura Pighi

Simone De Nitto

Martina Montagnana

Giuseppe Lippi ( $\nabla$ giuseppe.lippi@univr.it )

\section{Short Report}

Keywords: SARS-CoV-2, COVID-19, Omicron, Viral load, Infectivity

Posted Date: February 21st, 2022

DOI: https://doi.org/10.21203/rs.3.rs-1380500/v1

License: (9) This work is licensed under a Creative Commons Attribution 4.0 International License.

Read Full License

Version of Record: A version of this preprint was published at Journal of Infection on February 1st, 2022. See the published version at https://doi.org/10.1016/j.jinf.2022.02.025. 


\section{SARS-CoV-2 Omicron infection is associated with high nasopharyngeal viral load}

\section{Gian Luca Salvagno ${ }^{1,2}$, Brandon M. Henry ${ }^{3,4}$, Laura Pighi ${ }^{1,2}$, Simone De Nitto ${ }^{1,2}$, Martina Montagnana $^{1 \text { II }}$, Giuseppe Lippi ${ }^{1 \text { II }}$}

1. Section of Clinical Biochemistry, University of Verona, Verona, Italy

2. Service of Laboratory Medicine, Pederzoli Hospital, Peschiera del Garda, Italy

3. Disease Intervention \& Prevention and Population Health Programs, Texas Biomedical Research Institute, San Antonio, Texas, USA

4. Section of Clinical Biochemistry and School of Medicine, University of Verona, Italy.

${ }^{\text {II }}$ These authors share senior authorship in this work

Short title: Viral load of Omicron variant

Type of article: Short Note

\section{Corresponding author:}

Prof. Giuseppe Lippi

Section of Clinical Biochemistry

University Hospital of Verona

Piazzale L.A. Scuro, 10

37134 Verona - Italy

Tel. 0039-045-8122970

Fax. 0039-045-8124308

Email: giuseppe.lippi@univr.it 


\section{Abstract}

Background: We provide here direct comparison of viral load in nasopharyngeal specimens of patients infected by Omicron and former severe acute respiratory syndrome coronavirus 2 (SARS-CoV-2) variants.

Methods: We retrospectively reviewed the results of SARS-CoV-2 testing conducted during two corresponding periods of years 2021 and 2022 (i.e., between 3-9 January), characterized by local prevalence of Alpha (>95\%; January 2021) and Omicron (>90\%; January 2022) SARS-CoV-2 variants, respectively. In both years viral load was quantified using the same method and instrumentation (Seegene Allplex SARS-CoV-2 Assay).

Results: The total number of patients with positive SARS-CoV-2 tests was 47 in January 2021 and 118 in January 2022, respectively. The cycle threshold (Ct) values in January 2022 (median Ct value, 27.5; IQR, 23.5-32.7) when the Omicron variant was prevalent were significantly lower than those measured during the same period of the year 2021 (median $\mathrm{Ct}$ value, 31.8; IQR, 26.4-37.6; $\mathrm{p}=0.007$ ), when the Alpha variant was prevalent. The rate of subjects with high nasopharyngeal viral load was over 2-fold higher in January 2022 than in January 2021 (45/118 vs. 10/47; Odds ratio, 2.28 and 95\%CI, 1.03-5.03; $\mathrm{p}=0.041)$.

Conclusions: These results support the suggestion that aerosols released by patients infected by SARS-CoV-2 Omicron variant may contain higher viral particles than those released by subjects infected with previous strains.

Keywords: SARS-CoV-2; COVID-19; Omicron; Viral load; Infectivity 


\section{Introduction}

Previous evidence, such as that published by Zheng et al. [1], suggest that a high breath emission rate of viral particles may be one of the most important reasons for justifying the higher transmissibility of the new severe acute respiratory coronavirus 2 (SARS-CoV-2) Omicron variant. Unfortunately, no direct comparison of viral load with previous SARS-CoV-2 variants has been reported in the work of Zheng et al., so that this conclusion remains intriguingly speculative. In order to provide a direct comparison of the viral load in nasopharyngeal specimens of patients infected by Omicron and former SARS-CoV-2 variants, we retrospectively reviewed the results of SARS-CoV-2 testing conducted during two corresponding periods of years 2021 and 2022 (i.e., between 3-9 January), characterized by local prevalence of Alpha (>95\%; January 2021) and Omicron (>90\%; January 2022) SARS-CoV-2 variants, respectively.

\section{Materials and Methods}

The study population consisted of all patients with a positive SARS-CoV-2 test performed between 3-9 January 2021 or 2022, respectively. Routine nasopharyngeal samples were collected according to standardized practice in subjects referred to the Pederzoli Hospital of Peschiera del Garda (Italy) for undergoing routine SARS-CoV-2 testing for presenting symptoms of coronavirus disease 2019 (COVID-19) or for reporting close contact with SARS-CoV-2 positive subjects. In both these two years SARS-CoV-2 viral load was quantified using the same method and instrumentation (Seegene Allplex SARS-CoV-2 Assay; Seegene Inc., South Korea). This multiplex realtime polymerase chain reaction (PCR) assay detects four target genes of SARS-CoV-2 $(E, R d R P / S$ and $N)$ within a single sample, providing test results as cycle threshold $(\mathrm{Ct})$ values. Additional technical and analytical characteristics of this method are comprehensively described elsewhere [2]. The cumulative viral load that we measured 
in nasopharyngeal swabs was expressed as mean $\mathrm{Ct}$ value of the different SARS-CoV-2 genes. Final results were reported as median and interquartile range (IQR), and compared with Mann-Whitney or Chi square tests, when appropriate, using Analyse-it software (Analyse-it Software Ltd, Leeds, UK). This study was conducted as part of routine clinical laboratory operations, using pre-existing nasopharyngeal specimens collected for systematic SARS-CoV-2 diagnostic testing at the local facility, so that patient informed consent and Ethical Committee approval were unnecessary. All test results were anonymized before statistical analysis. The study was conducted in accordance with the Declaration of Helsinki, under the terms of relevant local legislation.

\section{Results}

The total number of patients with positive SARS-CoV-2 tests was 47 in January 2021 and 118 in January 2022, respectively, thus mirroring the dramatic surge of infections seen after local spread of the Omicron variant. Subjects with positive SARSCoV-2 tests in 2021 were significantly older (median age, 63 years; IQR, 47-80 years) compared to those testing positive in 2022 (median age, 42 years; IQR, 25-53 years; $\mathrm{p}<0.001$ ), whilst the sex distribution was similar (females: $68 \%$ in 2021 vs. $58 \%$ in 2022; $\mathrm{p}=0.107)$. The viral load measured with the Allplex SARS-CoV-2 Assay is reported in figure 1, showing that the $\mathrm{Ct}$ values in January 2022 (median $\mathrm{Ct}$ value, 27.5; IQR, 23.5-32.7) when the Omicron variant was prevalent were significantly lower than those measured during the same period of the year 2021 (median $\mathrm{Ct}$ value, 31.8; IQR, 26.4-37.6; $\mathrm{p}=0.007$, when the Alpha variant was prevalent. Importantly, the rate of subjects with high nasopharyngeal viral load (i.e., Ct values $<25$ ) was over 2-fold higher in January 2022 than in January 2021 (45/118 vs. 10/47; Odds ratio, 2.28 and 95\%CI, 1.03-5.03; $\mathrm{p}=0.041)$. 


\section{Conclusions}

In conclusion, the results of our retrospective analysis provide convincing support to the suggestion that aerosols released by patients infected by SARS-CoV-2 Omicron variant may contain higher viral particles than those released by subjects infected with previous SARS-CoV-2 strains, thus providing a solid biologic background to justify enhanced transmissibility of this new linage and higher prevalence of upper respiratory tract symptoms reported in other studies $[3,4]$.

\section{Declaration of Competing Interest}

The authors have no relevant competing interest to disclose in relation to this work.

\section{Funding}

None declared.

\section{Acknowledgments}

None.

\section{References}

1. Zheng J, Wang Z, Li J, et al. High amounts of SARS-CoV-2 in aerosols exhaled by patients with Omicron variant infection. J Infect. 2022 Feb 17:S01634453(22)00075-5. doi: 10.1016/j.jinf.2022.02.015. Epub ahead of print.

2. Liotti FM, Menchinelli G, Marchetti S, et al. Evaluation of three commercial assays for SARS-CoV-2 molecular detection in upper respiratory tract samples. Eur J Clin Microbiol Infect Dis 2021;40:269-277.

3. Migueres M, et al F, Raymond S, Lhomme S, Mansuy JM, Izopet J. Influence of immune escape and nasopharyngeal virus load on the spread of SARS-CoV-2 
Omicron variant. J Infect. 2022 Feb 7:S0163-4453(22)00054-8. doi: 10.1016/j.jinf.2022.01.036. Epub ahead of print.

4. Lippi G, Nocini R, Henry BM. Is SARS-CoV-2 Omicron (B.1.1.529) variant causing different symptoms? J Infect. 2022 Feb 17:S0163-4453(22)00077-9. doi: 10.1016/j.jinf.2022.02.011. Epub ahead of print. 
Figure 1. Nasopharyngeal viral load measured in subjects infected by severe acute respiratory syndrome coronavirus 2 (SARS-CoV-2) between 3-9 January 2021 (Alpha variant prevalent) and 3-9 January 2022 (Omicron variant prevalent).

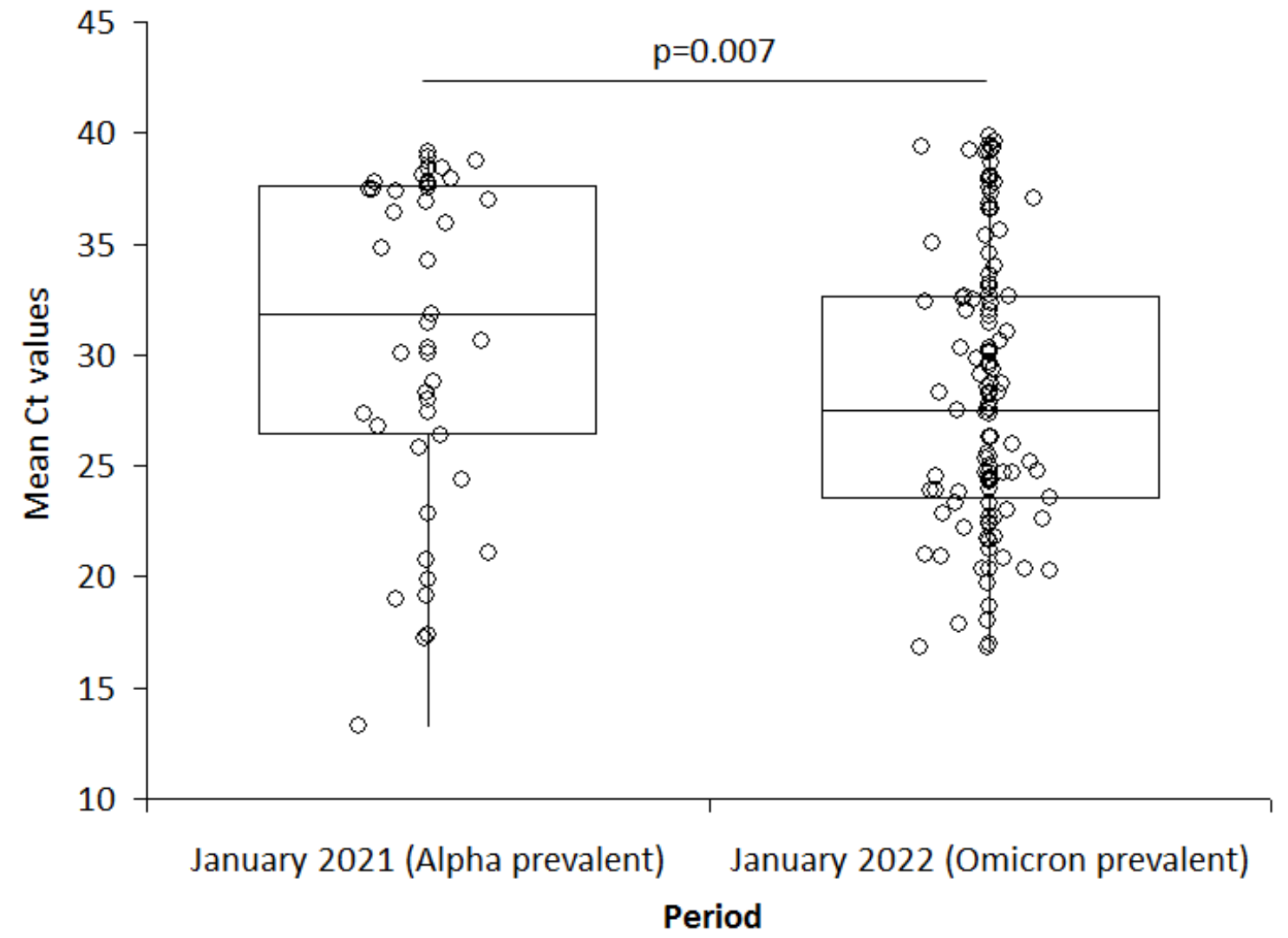

Ct, cycle threshold 